\title{
Trivium
}

Revue franco-allemande de sciences humaines et sociales - Deutsch-französische Zeitschrift für Geistesund Sozialwissenschaften

16 | 2014

La représentation politique

\section{Mise en scène et représentation de la politique aujourd'hui.}

\section{Ronald Hitzler}

Traducteur : Didier Renault

\section{OpenEdition}

Journals

Édition électronique

URL : http://journals.openedition.org/trivium/4776

ISSN : 1963-1820

\section{Éditeur}

Les éditions de la Maison des sciences de l'Homme

\section{Référence électronique}

Ronald Hitzler, « Mise en scène et représentation de la politique aujourd'hui. », Trivium [En ligne], 16 2014, mis en ligne le 01 mai 2014, consulté le 08 septembre 2020. URL : http:// journals.openedition.org/trivium/4776

Ce document a été généré automatiquement le 8 septembre 2020

\section{(c) (1) $\odot$}

Les contenus des la revue Trivium sont mis à disposition selon les termes de la Licence Creative Commons Attribution - Pas d'Utilisation Commerciale - Pas de Modification 4.0 International. 


\title{
Mise en scène et représentation de la politique aujourd'hui.
}

\author{
Ronald Hitzler
}

Traduction : Didier Renault

Nous remercions M. Ronald Hitzler de nous avoir accordé l'autorisation de traduire ce texte pour le présent numéro.

Wir danken Herrn Ronald Hitzler für die freundliche Genehmigung, diesen Artikel in französischer Übersetzung zu publizieren.

Quand nous parlons de représentation de la politique - pas exclusivement, mais tout au moins « de nos jours »- nous parlons en premier lieu et pour l'essentiel de la mise en scène des hommes politiques. Même si la reconstruction des règles et des régularités, des modèles et des structures, éventuellement aussi des effets et des conséquences des démarches de représentation de la politique ${ }^{1}$ 'est naturellement pas épuisée de cette manière, je me limiterai néanmoins ici pour l'essentiel à certains aspects des problèmes de l'action liés à la façon dont les politiciens professionnels se présentent eux-mêmes dans la démocratie représentative contemporaine. C'est en me concentrant sur ce thème que je me préoccuperai tout d'abord dans ce qui suit du phénomène de « la mise en scène de la politique " c'est-à-dire d'une forme de pratique de la " politique comme métier » qui vise avant tout à la popularité, aux effets sur le public, dans les conditions de possibilité et de contrainte de mise en scène impliquées par les mass-médias. Mais je m'attacherai également au phénomène de la représentation personnelle - quel que soit son genre - de ce que nous associons (selon les différents cas) au concept de "politique ", et pour le dire de manière générale, par conséquent, à la représentation « du politique ${ }^{2} »$. 


\section{I. «Faire de la politique » comme art de la mise en scène.}

2 Tout politicien (au moins dans une démocratie représentative) doit, s'il veut être élu et réélu, s'il veut construire et poursuivre sa carrière, s'efforcer de communiquer une impression de rayonnement personnel, d'expérience, de responsabilité, de culture, de conviction et de crédibilité. Et tout politicien doit dans la mesure du possible éviter de paraître bureaucratique, suffisant, arrogant, égoïste, avide de pouvoir, menteur, cynique et entêté. Autrement dit: tout homme politique est confronté au problème d'une mise en scène convaincante de l'autorité, du zèle, de la tolérance, de la fidélité à ses principes, de la compétence, de la présence, de l'humanité, et de tous les autres topoi positivement connotés du même genre, et est donc censé revendiquer la possession de qualités qui doivent - et peuvent aussi, manifestement - pousser d'autres hommes à le considérer parmi tous les autres comme le meilleur représentant et le meilleur avocat de leurs intérêts. Il s'agit en particulier d'apparaître comme une personnalité familière (connue) et digne de confiance (intègre).

3 C'est là, pour ainsi dire structurellement, la composante manipulatrice de la technique de l'agir politique. À elle seule, elle ne suffit manifestement pas à réussir en politique ${ }^{3}$. Elle doit d'abord et avant tout, être assortie d'un sens du "moment opportun», des courants et des tendances, de "l'esprit de l'époque», des réformes et des transformations qui «sont dans l'air ». "Celui à qui font défaut cette connaissance et cette pratique politiques générales ", écrit Agnès Heller, " peut bien être aussi raffiné qu'il le veut, feindre de la manière la plus rouée, et avoir recours aux instruments de la politique les plus appropriés, il ne deviendra cependant jamais un homme politique de premier plan ${ }^{4}$.»

4 Harold D. Laswell avait déjà lui-même révisé son concept initial de la personnalité typique de l'homme politique, celle d'une personnalité visant essentiellement le pouvoir ${ }^{5}$ en mettant bien plus fortement en relief l'importance du pragmatisme, de la souplesse et de la capacité à faire des compromis pour une carrière politique couronnée de succès ${ }^{6}$. Car l'homme politique, tout au moins (mais sans doute pas seulement) dans une démocratie représentative, et par opposition au bureaucrate par principe ancré dans de solides structures, occupe fondamentalement une position instable. Il lui faut par conséquent s'efforcer en permanence, dans des dimensions multiples, et en se tournant vers des publics divers, de devenir, d'être et de rester aimé $e^{7}$ Gore Vidal ${ }^{8}$ a désigné ces dispositions ou ces capacités qui font pour ainsi dire des hommes politiques des démocraties représentatives des opportunistes professionnels comme le talent particulier consistant à savoir infailliblement, par exemple, quand il faut partir et quand il faut rester, quand lâcher prise et quand se montrer intraitable.

5 Selon Vidal, un politicien dans une démocratie représentative doit être sociable, ou du moins donner l'impression qu'il l'est, sincère sans pourtant jamais dévoiler toutes les cartes qu'il a en main, et curieux des hommes, car son métier lui serait insupportable dans le cas contraire. Par ailleurs, un homme politique ne doit pas sembler intelligent à l'excès, il doit souvent sourire, tout en donnant en permanence une impression de sérieux, et ne doit pas paraitre hautain. Le politicien doit avoir un sens des situations, et quand on lui pose la bonne question, il doit toujours connaître la réponse qui semble «juste». Selon Christian von Krockow, l'activité politique de nos représentants du peuple consiste typiquement à «intriguer, affirmer, s'imposer dans les travaux des 
commissions [...] l'éloquence [...] la présence [...] le sang-froid en cas d'imprévu et la maîtrise des règles du jeu [...] la faculté d'adaptation et l'obligeance [...] la capacité à nouer des liens, et, si nécessaire, de les défaire à nouveau [...] se rendre utile. Travailler assidûment, ou tout au moins paraître très affairé9."

6 L'essentiel est par conséquent l'apparence que l'homme politique possède de «bonnes " qualités, et non leur présence réelle. Machiavel est même allé «jusqu'à affirmer qu'elles sont nuisibles lorsqu'on les possède et qu'on en fait un usage constant, et qu'elles sont utiles quand on ne fait que se donner l'apparence de les posséder ${ }^{10}$.» Du point de vue de la «logique » du politique, le problème de l'action bonne ou mauvaise revient à savoir si les moyens mis en œuvre étaient appropriés ou non.

7 Il en résulte que ce qui fait la réussite de l'homme politique, c'est d'agir selon la rationalité des fins et des moyens, indépendamment des positions philosophiques : car la politique en tant que telle est précisément, aux yeux de Machiavel, l'art de discerner les conditions générales socio-historiques, de les évaluer correctement et de réagir « judicieusement » à leur égard, autrement dit de les utiliser dans chaque cas au profit des buts que l'on se propose, ou encore de mettre ses buts en accord avec elles, et de s'efforcer ainsi« de participer du pouvoir ou d'influencer sa répartition ${ }^{11}$ ». Réussir en tant que politicien signifie donc maîtriser cet art et agir autant que possible sans illusions, qu'elles portent sur soi-même ou sur les autres. Et cela implique donc de nos jours, dans une collectivité organisée sous forme de démocratie représentative, de faire naître, en particulier chez «les principaux intéressés ", l'impression qu'ils bénéficient de la mise en œuvre d'une (certaine) politique de sorte qu'ils aient davantage intérêt à ce que l'on continue de l'appliquer, plutôt que de voir d'autres hommes mettre en œuvre une (autre) politique. Ce que l'on pourrait encore désigner de nos jours, dans les collectivités régies par la démocratie représentative, comme « pouvoir politique » naît ainsi avant tout d'une aptitude à la présentation ${ }^{12}$.

8 Ce qui apparaît ainsi, c'est une identité poussée entre "politique-spectacle » et action politique réell ${ }^{13}$. Le spectacle est la politique, pourrait-on dire en exagérant à peine. Cela signifie que l'homme politique travaille en permanence - du moins en même temps que le reste - à son «image »: il s'efforce en chaque circonstance, face à différents interlocuteurs donnés, et dans des circonstances déterminées, de produire un «effet » tel qu'ils auront de sa personne ou de sa personnalité l'impression qu'il souhaite éveiller chez eux ${ }^{14}$. Cependant, si l'on considère les éléments dramaturgiques de l'existence des politiciens comme structurant, pour ainsi dire, leur action ${ }^{15}$, on risque de céder à la tentation d'hypostasier la totalité de ce que l'on a sous les yeux comme le résultat d'une stratégie délibérée. L'homme politique n'apparaîtra plus alors, aux yeux du spectateur soupçonneux, que sous les traits du mime talentueux et du metteur en scène roublard, du mystérieux marionnettiste et de l'intrigant, du tacticien rusé et, avant tout, précisément, du machiavélien au sang froid. De telles aptitudes et de tels dons sont sans doute essentiels pour la survie dans l'univers de significations de la politique : qui recherche le succès dans ce domaine ne peut faire autrement que de se tenir en permanence dans une position d'attente calculatrice, tablant sur le fait que chacun (lui-même y compris) n'a en dernier ressort d'autre choix que de se faire valoir aux dépens de son ou de ses semblables, quels qu'ils soient. L'essentiel de ce que fait le politicien, lorsqu'il fait de la politique, est cependant moins la stratégie individuelle d'un acteur que l'habitus collectif d'une profession. Autrement dit, quand il agit, c'est souvent plus «inconsciemment» qu'il n'en donne l'impression - se pliant à des 
routines propres à son milieu, à des règles de vie typiques des groupes auxquels il appartient, aux conventions de subcultures. Quand il essaie de nous en "faire accroire ", il ne fait souvent que répéter ce que d'autres lui font croire. Il joue alors son rôle, plus souvent que ne le pense le critique du café du commerce, tout à fait dans le sens de Georg Simmel "non comme une hypocrisie et une duperie, mais comme l'irruption de la vie personnelle dans une forme d'expression qu'il trouve devant lui préexistante et prédéfinie, sous une forme ou sous une autre ${ }^{16}$. "

9 Les techniques de mise en scène de toutes sortes sont donc avant tout, dans le «milieu» politique (professionnel), expérimentées, intégrées comme habitudes, transmises, et même en un sens institutionnalisées, de telle sorte que typiquement, les hommes politiques sont en général, à un degré bien supérieur aux «hommes normaux ", des virtuoses de l'intervention stratégique et de la combinaison stratégique de formes d'expression diverses: «L'art de la politique se voit de la sorte pour ainsi dire objectivé, et devient en un sens indépendant de la contingence de l'auteur de l'action, et même du degré de son talent ${ }^{17}$. » La technique d'impression management « en toute situation " est en quelque sorte une pure et simple question de survie pour tout homme politique. Et avant tout, son impact médiatique est de nos jours « le pain » dont il se «nourrit» pour la gestion de sa carrière ${ }^{18}$. Les politiciens "s'exposent », il leur faut s'exposer, ils doivent être présents, avant tout dans les médias, parce que de cette présence dépend leur notoriété, qui est à son tour une condition essentielle de leurs chances de rester "en jeu» autrement dit de se représenter et d'être réélus, et (idéalement) de continuer à progresser dans la hiérarchie politique.

10 On est ainsi tenté de partir du principe que - d'une perspective dramatologique «faire de la politique» professionnellement ou quasi professionnellement se joue moins dans le cadre de catégories de mesures appropriées ou non que dans celui de mises en scène réussies ou non. De ce point de vue, «faire de la politique » vise moins une action appropriée pour atteindre des objectifs positifs pour la collectivité qu'à désigner, expliciter, évaluer et légitimer des objectifs déterminés, en comparaison avec d'autres objectifs. De telles démarches de légitimation font naturellement partie des tâches de routine du politicien de métier, du moins dans la démocratie représentative (actuelle). Il en résulte que l'exercice quotidien du politicien apparaît bien moins comme une pratique instrumentale que comme une pratique expressive ou symbolique : ce dont il s'agit avant tout pour lui, quotidiennement, c'est d'avoir et d'entretenir des contacts personnels rigoureusement à tous les niveaux de la hiérarchie sociale : des relations institutionnelles et organisationnelles directes et indirectes, des groupes "de référence ", c'est-à-dire des contacts avec des électeurs, des lobbyistes, des membres de son parti, mais aussi des membres d'autres partis, des collègues, des collaborateurs, etc.

11 Les données «objectives", les chiffres, les faits, les mémorandums, documents et projets de lois paraissent, sous cet aspect rituel, dans le sens de Luckmann ${ }^{19}$ de l'agir politique différencié institutionnellement, très secondaires comparativement à ce savoir (spécifique) « impalpable», qui résulte d'une multitude d'indications et de mises en garde informelles, de discrétions et d'indiscrétions, d'humeurs et d'intuitions, de spéculations et d'intimités, d'opinions et de préjugés, d'évidences individuelles et collectives. Les sources de la construction du sens politique semblent être (au grand dam de nombreux « conseillers » qui confondent la politique et les sciences politiques) davantage des communications "entre les lignes», des canaux non officiels, des 
confidences au téléphone, "en passant ", en marge de réunions, que des statistiques et des analyses scientifiques, des rapports d'experts et des bases de données. Au premier chef, l'homme politique ne donne pas forme (contrairement à l'apparence volontiers évoquée) aux processus de décision politique, mais il interprète et «explique » des décisions actuelles, déjà prises ou à venir, et leur confere ainsi la dignité du normatif ${ }^{20}$. Aux yeux du citoyen normal, il apparaît ainsi typiquement (et souvent à tort) comme un expert des questions et des problèmes politiques.

Le dilemme de ce que l'on appelle la " classe politique ${ }^{21}$ » dans son ensemble consiste cependant précisément en ce qu'elle se légitime aux yeux du public en prétendant qu'elle est en mesure de dépasser, voire d'éliminer (en dernière instance) les conflits et les contradictions d'origine sociale dans le système politique qu'elle "dirige", alors qu'elle ne peut jamais, en fait, que produire des moyens (plus ou moins adéquats) pour les « organiser ${ }^{22}$ ». On est donc tout naturellement amené à se demander pour quelle raison les politiciens ne cessent de répéter cette promesse chimérique, perpétuant ainsi la fiction d'une capacité à résoudre les problèmes dont ils sont en fait dépourvus, tandis que dans le même temps, et de manière croissante, ils nient explicitement et expressément l'idée du pouvoir ${ }^{23}$.

13 Tandis que d'autres approches dramatologiques ${ }^{24}$ de la sociologie politique s'efforcent traditionnellement d'expliquer cette incohérence par un modèle de «l'avant-scène » et des « coulisses », je fais ici délibérément abstraction de la question analytique de savoir comment le système politique fonctionne réellement ${ }^{25}$. Je pars au contraire de l'hypothèse que s'il existe des "coulisses " du pouvoir "réellement» secrètes, entièrement passées sous silence et hermétiquement closes, je n'en sais "réellement " rien pour ma part, et même, à strictement parler, que je ne peux rien en savoir « réellement». Par conséquent, l'objet de mon intérêt de connaissance pour cette question se limite strictement à la dimension visible ${ }^{26}$ et à la compréhension que les acteurs ont d'eux-mêmes sous cet aspect. Néanmoins, la comparaison entre la première et la seconde s'avère être une construction manifestement précaire et toujours plus menacée. La transformation de la société souvent constatée dans les débats actuels sur la modernisation s'accomplit également dans le système fonctionnel de la politique, même si les formes qu'elle y prend peuvent dissimuler les événements réels jusqu'à les rendre parfois impossibles à identifier.

\section{Sur la construction politique de la réalité.}

Des experts scientifiques de ce domaine ${ }^{27}$ tendent à interpréter tout acte de représentation politique - pas uniquement, mais bien sûr en premier lieu ceux des politiciens professionnels - comme un acte communicationnel persuasif, autrement dit visant à convaincre ou à emporter l'adhésion: "La compétence communicationnelle est nécessaire à l'homme politique moderne. Il lui faut disposer d'une capacité à transmettre la politique, et plus il sait utiliser en virtuose le registre de la symbolique politique, plus il aura de chances de succès ${ }^{28}$. $\gg$ On pourrait aussi dire que même dans des périodes où il est presque impossible de surestimer l'importance des images ${ }^{29}$ (animées) dans la totalité des processus de construction de la réalité - donc également et au premier chef dans le processus politique -, du point de vue dramatologique, l' élément fondamental de la politique est néanmoins (en dernière instance) la langue, ou pour mieux dire : le discours, la langue appliquée. Ce qui constitue la langue de (dans) la 
politique va des mémos et des discussions informelles lors d'allocutions, de débats, des conférences de presse jusqu'aux rassemblements de masse et aux idéologies générales. La langue de (dans) la politique est pour ainsi dire au centre de ce que l'on pourrait nommer l'impression management politique, c'est-à-dire la démarche consistant à donner une image de soi propre à persuader les autres de faire d'eux-mêmes ce que l'on souhaite qu'ils fassent. On pourrait aussi dire: la politique est, en un sens, l'art de remplacer des confrontations physiques par des confrontations de signes ou de symboles. De ce point de vue, il faut néanmoins remarquer que par principe, et contrairement à une vision naïve de l'importance de l'échange de points de vue entre hommes politiques ou entre camps politiques, il ne s'agit pas pour le politicien, ou seulement dans des cas extraordinairement rares, de convaincre de quelconques "adversaires" de la justesse de sa position, autrement dit de les amener à changer d'avis sur un sujet donné. La rhétorique politique sert plutôt, avant tout, à encourager les adhérents, à stimuler les sympathisants, à rallier les indécis, ainsi qu'à faire taire les critiques dans son propre camp. Elle sert aussi, dans une large mesure, à apaiser le public (en donnant des explications, en désignant des responsables, en s'excusant), à argumenter pour ou contre certaines positions, à légitimer ou anéantir des idées politiques et des visions du monde, à se mettre en valeur et à stigmatiser des adversaires ou des rivaux, à prêter une résonance émotionnelle à des faits bruts, à évoquer des «images " positives ou à rectifier des « images ${ }^{30}$ » négatives, à produire l'assentiment et les actions désirées (ou souhaitées), etc.

Il semble effectivement qu'existe quelque chose comme des "grammaires ${ }^{31}$ " d'une mise en scène politique réussie; des grammaires qui ne sont en aucune manière seulement propres à être appliquées, par des spécialistes par exemple, ex post, aux séquences et aux contextes factuels de l'action, et dont tout acteur politique dispose en bonne partie au moins sous la forme d'une " connaissance des recettes ${ }^{32}$ » usuelle, mais que le politicien professionnel informé qui vise au succès et avant tout les conseillers en image et les experts en marketing connaissent sur le bout des doigts et utilisent de manière stratégique ${ }^{33}$.

Toutes ces techniques et subtilités, tous ces artifices relèvent à mes yeux du phénomène de la mise en scène de la politique. La mise en scène de la politique sert avant tout à exister et à l'emporter dans la lutte concurrentielle avec d'autres prétendants à la faveur du public. Il est néanmoins clair qu'un talent d'acteur ou une maitrise des médias consommés mais purement orientés techniquement ne suffisent pas - même assortis d'un "flair» pour "l'esprit de l'époque» - à faire preuve de manière convaincante et durable que l'on est un représentant légitime du système politique. La politique, et par conséquent l'homme politique individuel, doit au contraire contribuer à représenter ce qui ne peut trouver une expression que dans et par le biais de la politique: la représentation d'une certaine réalité qui transcende le quotidien, la représentation du monde « idéal » d'une organisation donnée de la collectivité, ou plus précisément: une idée de la collectivité acceptée et réclamée par (au moins) une " clientèle " déterminée ${ }^{34}$. Autrement dit, celui qui incarne avec succès le système politique doit être en mesure de faire valoir - pour ainsi dire à tous les niveaux - qu'il représente ce que son électeur considère comme essentiel pour la collectivité. Du point de vue dramatologique, il s'agit par conséquent moins de posséder un tel motif ou de se conformer effectivement à une telle idée que de savoir persuader (au moins une 
«clientèle » déterminée) que c'est bien cette idée qui inspire la volonté (politique) propre du représentant.

Indépendamment du fait que l'homme politique croie ce qu'il dit, voire même dise ce qu'il croit, il lui faut pour réussir agir en fonction d'un certain nombre de règles immanentes de la politique. Une partie importante et souvent commentée de ces règles, pas seulement, mais avant tout dans les démocraties représentatives modernes comme les nôtres, est de rendre plausible l'idée qu'il veut le bien « de tous » (du moins de tous ceux auxquels il s'adresse) et que c'est pour cela que l'on doit lui confier le pouvoir ou lui permettre de le conserver. Néanmoins, une autre partie de ces règles bien moins souvent évoquée dans nos sociétés modernes structurellement pluralistes dans des circonstances normales -implique qu'il soit capable de mettre en œuvre le motif de certains pouvoirs et idées "d'un ordre plus élevé » au service de ses objectifs. C'est ce que confirme par exemple Herfried Münkler dans son commentaire de Machiavel, lorsqu'il écrit qu'appartiennent aussi aux "moyens immanents de la politique [...] l'apparence de la transcendance, et l'utilisation tactique habile de cette apparence ». C'est ainsi qu'une religion comprise un sens intégralement fonctionnel dans le sillage de Luckmann - comme "attribuant du sens par la mythologisation ", contribue de manière essentielle à la stabilisation de n'importe quel ordre, fût-il résolument athée. Revêtir d'un habit religieux, quelle que soit sa nature, la facticité de la domination lui confère la dignité du normatif. Car toute domination qui vise à rester stable doit s'entourer d'un « liseré de croyance ", comme le pensait déjà Max Weber ; ce qui signifie être légitimée par des références transcendantes : « Les notions de légitimité ne fondent pas [...] les relations de domination, mais elles imprègnent la supériorité effective et lui confèrent le rang d'une relation sociale admise pour de bonnes raisons $^{35}$.» Le peuple, selon Machiavel, a besoin de l'opium de la religion afin de pouvoir obéir ; l'État, la collectivité, à besoin d'un peuple obéissant pour rester stable ; il en résulte que l'État a besoin de la religion ${ }^{36}$.

Dans ce sens dérivé, l'homme politique en tant que tel représente donc - dans l'idéal effectivement la collectivités ${ }^{37}$. Il parvient à persuader les citoyens qu'il est l'incarnation de cet État dont il a par ailleurs su les convaincre qu'il était le garant de leur bienêtre $^{38}$. La représentation ainsi comprise sert entre autres, intentionnellement, ou comme c'est sans doute plus fréquemment le cas, incidemment, à lier les membres de la société à un principe de domination en vigueur. Selon Alfred Schütz ${ }^{39}$, elle est au service de l'objectif utile qui consiste à unifier les gouvernés. Pour Siegfried Landshut ${ }^{40}$, " ce qui est à l'œuvre de manière présente, tangible, dans la représentation ", c'est une idée de la conduite de vie qui s'impose à tous, « ce principe particulier qui fait l'unité et la communauté du vivre ensemble politique, un principe régulateur qui joue le rôle d'un impératif de conduite de vie ${ }^{41}$. »

L'homme politique per se - et donc au-delà de ses inclinations personnelles ou partisanes, de ses propres attentes, intérêts ou objectifs - possède donc toujours également une fonction productrice de sens, une fonction symbolique de garant de la réalité. Pour le dire autrement : l'homme politique «idéal » qui se présente comme tel, apparaît selon cette fonction générale consistant à représenter l'idée du "système " politique dans l'absolu comme un médium, comme un intermédiaire de ce registre de la réalité de l'organisation des collectifs sociaux qui vise à s'imposer, qui transcende la réalité quotidienne, mais n'en revêt pas moins une importance considérable pour la vie en commun au quotidien. L'homme politique assume une fonction de représentation $d u$ 
citoyen, mais exerce aussi vis-à-vis de lui une sorte de fonction sacerdotale séculaire : à l'égard de ceux dont il est formellement le "représentant ", il fait office, en fait, de représentant d'une idée ou d'un corpus d'idées ${ }^{42}$.

Pour être convaincant, il lui faut cependant « être un maître dans l'art de simuler et de dissimuler ${ }^{43}$ " car ses propres manières d'agir, s'il veut leur assurer le succès, ne doivent justement pas s'en tenir au cadre des normes que l'homme politique entend imposer au citoyen, et qu'il prétend, qu'il doit prétendre respecter lui-même ${ }^{44}$. Alors que le citoyen normal est captif de mythes de toute espèce sur " Dieu et le monde », tandis que sa vie lui paraît régie par des puissances et des forces invisibles, l'homme politique doit garder "les idées claires » et être en mesure de déchiffrer des situations et des conjonctures complexes. Ce n'est que de cette manière qu'il peut non seulement vivre dans la collectivité et y avoir sa place, mais aussi la diriger, anticiper, orienter, contrôler les évolutions, car lorsque des défaillances de l'État « ne sont pas reconnues et qu'on les laisse se répandre au point que chacun peut les voir, il n'y a alors plus de remède ${ }^{45}$. "

21 L'homme politique concentre ainsi d'une certaine manière dans sa personne, dans ses actions et objectivations, des séquences et des enchaînements politiques complexes. Il est celui dont « on » attend qu'il soit en mesure de révéler, d'interpréter, d'expliquer, et effectivement : de rendre plausible le sens - pas seulement fortuitement dissimulé - de mesures d'organisation sociale. Les modèles d'interprétation que fournit l'homme politique - s'il n'est pas le seul à le faire, il joue ici un rôle particulier puisqu'au-delà des mass-médias, il les présente aussi en face-to-face - ont pour fonction, par-delà toutes les mises en scène visant ou suivant les intérêts de parti, de représenter symboliquement une conception de la réalité (politique) en tant que telle. De cette manière, en tant que représentant, l'homme politique amène à " une effectivité actuelle ce qui existe, mais ne serait pas «là » sans lui, ne pourrait avoir d'effets en tant que phénomène visible, [...] une dimension idéelle, spirituelle ${ }^{46}$. " Par principe, et l'on pourrait dire inévitablement, le représentant représente aussi l'idée du politique en tant que totalité.

Autrement dit: le représentant politique, en tant que tel, transmet au citoyen une interprétation politique du monde acceptable, qui, idéalement, ne risque d'être remise en cause dans ses orientations fondamentales ni par un changement de personnes, ni par un changement de cap politique ${ }^{47}$. Le représentant politique, à titre d'élément de la symbolique politique en tant que telle, contribue à faire considérer un registre donné d'interprétations politiques de la réalité comme adéquat et pertinent ${ }^{48}$.

\section{Sur le contenu symbolique du politique.}

Considéré sous l'angle sémiotique, le représentant correspond, selon Husserl ${ }^{49}$, à une représentation de substitution artificielle, symbolique. Il tient lieu de quelque chose pour quelque chose en vue de quelque chose. Il est un symbole établi sous forme emblématique $^{50}$. Qui perçoit le représentant comme phénomène significatif, et même comme porteur de significations mystérieuses, perçoit sans doute en même temps quelque chose d'aussi complexe qu'un être humain (tout au moins un certain type d'être humain, peut être même un Autrui significatif hautement individualisé). Mais la dimension essentielle pour un représentant est par définition (et naturellement) sa fonction référentielle. Le représentant nous apparait comme l'un des éléments d'une chaîne d'associations dont le maillon qui nous est présenté appartient à un registre de 
la réalité transcendant la vie quotidienne, c'est-à-dire celui de l'ordre des collectifs sociaux ${ }^{51}$.

Cette fonction de représentation se rattache ainsi presque automatiquement au rôle de l'homme politique de métier qui requiert une mise en scène. En un mot: on n'est pas homme politique, à la suite de quoi on choisirait de représenter ou de ne pas représenter, de représenter plus ou moins. Au contraire, on représente constamment parce qu'on est un homme politique. Être représentant est pour ainsi dire l'essence, l'incarnation de la responsabilité symbolique - pas uniquement, mais aussi et précisément en tant qu'homme politique ${ }^{52}$. Parce que le représentant n'est pas simplement un symbole emblématique, mais parce qu'il agit en tant que tel, il n'est donc pas seulement une part du système sémiotique politique, mais aussi une part du processus sémiotique, de ce déroulement communicationnel (médiatique) qui transmet à l'observateur usuel de ce scénario le sens sous-jacent de la politique. En bref: le représentant, en tant que référentialité symbolique est part d'un rituel politique qui constitue lui-même un élément d'une culture politique donnée. L'idée du politique, qui transcende la vie quotidienne, se matérialise, ou pour le dire métaphoriquement s'incarne au jour le jour dans le représentant, qui, dans sa pure dimension perceptible, ne renvoie pas à lui-même (quel que soit ce « lui-même ») mais, au-delà de lui-même, à l'autre réalité de l'ordre idéal des collectifs sociaux.

Le représentant a donc sans aucun doute un sens subjectif - d'une part pour celui qui est chargé de l'incarner, d'autre part pour celui qui le perçoit. Il a en outre tout aussi indéniablement une signification conjoncturelle, selon l' "arène " spécifique et les conditions spécifiques préalables à son apparition. Mais il a aussi - en tant que signe, ou que symbole significatif - un sens objectif ${ }^{53}$. Et ce sens objectif tient essentiellement à sa fonction rituelle, à son incarnation de l'ordre politique d'une société considéré comme légitime. Car, comme l'avait déjà noté le romantique Friedrich von Hardenberg mieux connu sous son pseudonyme Novalis, « Le souverain mystique n'a-t-il pas besoin, comme toute idée, d'un symbole, et quel symbole serait plus digne et plus approprié qu'un homme aussi aimable que remarquable ${ }^{54}$ ?»

Ce dont il est question ici, que l'on pourrait désigner comme le potentiel charismatique de l'homme politique notable consiste donc avant tout selon cette conception- au-delà de ses inclinations personnelles ou partisanes, de ses propres attentes, intérêts ou objectifs - à faire office de représentant dans cette acception et par conséquent de faire preuve en particulier d'une compétence symbolique, une compétence à instaurer sens et signification et à se faire le garant de la réalité. Idéalement, il serait pour le citoyen normal un médium, un médiateur vers ce domaine de la réalité du politique qui transcende l'expérience du quotidien, mais n'en revêt pas moins une importance considérable dans la vie quotidienne de la société. Idéalement, il serait à l'égard de celui qu'il représente formellement également le représentant d'une idée, ou d'un corpus d'idées ${ }^{55}$. Idéalement, il concentre d'une certaine manière dans sa personne, dans ses actes et dans ses objectivations, des contextes et des déroulements politiques complexes. Idéalement, nous devrions pouvoir attendre de lui qu'il soit capable d'élucider, d'interpréter, d' "expliciter» le sens - pas seulement fortuitement dissimulé - de mesures d'organisation sociale. Car s'il pouvait faire preuve de ces qualités, l'homme politique professionnel contemporain parviendrait précisément à démontrer plausiblement, dans l'idéal, qu'il est certes "un homme parmi tous les 
autres ", mais néanmoins justement le meilleur (relativement) 'de tous', au moins de tous ceux qui sont disponibles pour le poste qu'il occupe ou qu'il brigue.

Ce qui prouve néanmoins que la majorité du personnel politique contemporain peine à donner l'impression d'une compétence propre à répondre aux préoccupations de la collectivité dans une période ressentie comme difficile, c'est le jugement prédominant dans l' "opinion publique» - et cela depuis longtemps, et en dépit des "vogues momentanées » des protagonistes (et antagonistes) d'un certain nombre d'attitudes politiques en temps de guerre et de crise $^{56}$ - selon lequel la sphère politique établie serait totalement à court de visions programmatiques et d'inventivité idéologique pour la transformation et la réorientation de la société, et que les politiciens élus se caractérisent avant tout par leur avidité, pour eux-mêmes ou le lobby qu'ils représentent ${ }^{57}$. C'est ainsi que parmi bien d'autres choses, le catalogue - que le présent article expose lui aussi une fois de plus -des qualités prometteuses de succès (électoraux), qu'il s'agit de rendre crédibles, un catalogue qui se compose de comportements observés aussi bien que de déclarations d'hommes politiques, se condense pour l'acteur individuel sur la scène de la politique institutionnalisée sous la forme d'un livre de recettes, comme un programme de représentation de soi-même dont on pourrait dire qu'il est chroniquement sous-réalisé.

\section{BIBLIOGRAPHIE}

Arnim, H. v. (1993), Der Staat als Beute, Munich : Knaur.

Atkinson, M. (1984), Our Masters' Voices, Londres/New York : Methuen.

Beck, U. (1986), Risikogesellschaft, Francfort-sur-le-Main : Suhrkamp.

Beck, U./ Giddens, A./ Lash, S. : Reflexive Modernisierung, Francfort-sur-le-Main : Suhrkamp.

Beck, U./ Hajer, M./ Kesselring, S. (éd.), Der unscharfe Ort der Politik, Opladen : Leske + Budrich.

Berger, P./ Luckmann, T. (1969), Die gesellschaftliche Konstruktion der Wirklichkeit, Francfort-sur-leMain : Fischer.

Beyme, K. v. (1993), Die politische Klasse im Parteienstaat, Francfort-sur-le-Main : Suhrkamp.

Boissevain, J. (1978), Friends of Friends. Networks, Manipulators, and Coalitions, Oxford : Basil

Blackwell.

Boorstin, D. J. (1987), Das Image, Reinbek : Rowohlt.

Bourdieu, P. (1986), « Delegation und politischer Fetischismus », Ästhetik und Kommunikation, 61-62.

Dieckmann, W. (1981), Politische Sprache, politische Kommunikation, Heidelberg : Winter.

Dörner, A. (2000), Politische Kultur und Medienunterhaltung, Constance : Universitätsverlag Konstanz.

Dörner, A. (2001), Politainment, Francfort-sur-le-Main : Suhrkamp. 
Edelman, M. (1988), Constructing the Political Spectacle, Chicago : The University of Chicago Press.

Eulau, H./ Karps, P.D. (1977), « The Puzzle of Representation : Specifying Components of Responsiveness », Legislative Studies Quarterly, II, No. 3.

Ferber, C. v. (1970), Die Gewalt in der Politik, Stuttgart : Kohlhammer.

Foucault, M. (1978), Dispositive der Macht, Berlin : Merve.

Freyer, H. (1986) [1938], Machiavelli, Weinheim : Acta Humaniora.

Geißner, H. (1969), Rede in der Öffentlichkeit, Stuttgart : Kohlhammer.

Goffman, E. (1969), Wir alle spielen Theater. Die Selbstdarstellung im Alltag, Munich : Piper.

Goffman, E. (1971), « Techniken der Imagepflege », in : Id. : Interaktionsrituale, Francfort-sur-leMain : Suhrkamp, p. 10-53.

Gräber, D. A. (1976), Verbal Behavior and Politics, Urbana : University of Illinois Press.

Haseloff, O.W. (1969), « Über Symbolik und Resonanzbedingungen der politischen Sprache », in :

K. D. Hartmann (éd.) : Politische Beeinflussung, Francfort-sur-le-Main : Europäische Verlagsanstalt.

Heller, A. (1982), Der Mensch der Renaissance, Cologne-Lövenich : Hohenheim.

Hitzler, R. (1994), « Die banale Seite der Macht », in : Berking, H./Hitzler, R./ Neckel, S. (éd..) :

Politikertypen in Europa, Francfort-sur-le-Main : Fischer, p. 280-295.

Hitzler, R. (1996), « Die Produktion von Charisma », in : Imhof, K./Schulz, P. (éd.): Politisches

Raisonnement in der Informationsgesellschaft, Zurich : Seismo, p.. 265-288.

Hitzler, R. (1997), « Politisches Wissen und politisches Handeln », in : Lamnek, S. (éd.) : Soziologie und Politische Bildung, Opladen : Leske + Budrich, p. 115-132.

Hitzler, R. (1999), « Gewalt als Tätigkeit », in : Neckel, S./Schwab-Trapp, M. (éd.) : Ordnungen der Gewalt, Opladen: Leske + Budrich, S. 9-19.

Hitzler, R. / Kliche, T. (1995), « Zwischen Sozialtechnologie und Heiligkeit : Symbolpolitik und Symbolisierende Politik », Zeitschrift für Politische Psychologie,. 3, (4), p. 359-384.

Hofmann, G. (1982), « Politik als Beruf : Bonner Beobachtungen », in : Raschke, J. (éd.): Bürger und Parteien, Opladen : Westdeutscher, p. 55-68.

Husserl, E. (1970), « Zur Logik der Zeichen (Semiotik) », in: Id. : Philosophie der Arithmetik (Husserliana XII), La Haye : Nijhoff.

Ichheiser, G. (1927), « Die Antinomie zwischen Politik und Moral nach Machiavelli », Zeitschrift ftir Völkerpsychologie und Soziologie, p. 294-309.

Jarren, O. (2001), « 'Mediengesellschaft' - Risiken für die politische Kommunikation », Aus Politik und Zeitgeschichte, 41-42, p. 10-19.

Krockow, C. v. (1989), Politik und menschliche Natur, Munich : Deutscher Taschenbuch Verlag.

Landshut, S. (1964), « Der politische Begriff der Repräsentation », in: Ortlieb, H- D. (éd.) : Hamburger Jahrbuch für Wirtschafts- und Gesellschaftspolitik, Tübingen : Mohr (Siebeck).

Laswell, H. D. (1951), « Psychopathology and Politics » in : Id.: The Political Writings, Glencoe : Free Press.

Laswell, H. D. (1954) ,The Selective Effect of Personality on Political Participation » in : Christie, R./ Jahoda, M. (éd.) : Studies in the Scope and Method of the 'Authoritarian Personality', Glencoe : Free Press. 
Leibholz, G. (1958), Strukturprobleme der modernen Demokratie, Karlsruhe : C.F. Müller.

Leif, T. (2001), « Macht ohne Verantwortung », Aus Politik und Zeitgeschichte, 41-42, p. 6-9.

Leif, T./ Legrand, H.-J./ Klein, A. (éd.) (1992), Die politische Klasse in Deutschland, Bonn/Berlin : Bouvier.

Lipp, W. (1985), Stigma und Charisma, Berlin: Reimer.

Luckmann, T. (1985), « Riten als Bewältigung lebensweltlicher Grenzen », Schweizerische Zeitschrift für Soziologie, 3, p. 535-55.

Luckmann, T. (1991), Die unsichtbare Religion, Francfort-sur-le-Main : Suhrkamp.

Machiavelli, N. (1972), Der Fürst, Stuttgart : Kröner.

Marx, P./Pankoke, E. (1992), « Publizität und Enthusiasmus », in : Gauger, J.-D./ Stagl, J. (éd.) :

Staatsrepräsentation, Berlin : Reimer, p. 89-104.

Mayntz, R./ Neidhardt, F. (1989), «Parlamentskultur : Handlungsorientierung von

Bundestagsabgeordneten », Zeitschrift fur Parlamentsfragen, 3, p. 370-387.

Mayntz, R./ Scharpf, F. (1973), Planungsorganisation, Munich : Piper.

McGinnis, J. (1970), The Selling of the President, Harmondsworth : Penguin.

Meyer, T. (1992), Die Inszenierung des Scheins, Francfort-sur-le-Main : Suhrkamp.

Meyer, T. (2001), Mediokratie, Francfort-sur-le-Main : Suhrkamp.

Münkler, H. (1984), Machiavelli, Francfort-sur-le-Main : Suhrkamp.

Nedelmann, B. (1986), « Das kulturelle Milieu politischer Konflikte » in: Neidhardt, F./ Lepsius, M. Rainer/ Weiß, J. (éd.): Kultur und Gesellschaft. Sonderheft 27 der Kölner Zeitschrift für Soziologie und Sozialpsychologie, Opladen : Westdeutscher Verlag, p. 397-414.

Patzelt, W. (1991), « Das Amtsverständnis der Abgeordneten », Aus Politik und Zeitgeschichte, 21/22, p. 25-37.

Pesch, V. (2000), Handlungstheorie und Politische Kultur, Wiesbaden : Westdeutscher Verlag.

Price, C.M./ Bell, C.G. (1970), « The Rules of the Game », Journal of Politics, 32, p. 839-855.

Radunski, P. (1980), Wahlkämpfe, Munich/Vienne : Olzog.

Rapp, U. (1973), Handeln und Zuschauen, Darmstadt/Neuwied : Luchterhand.

Randow, J. (1962), «Studien zur Rhetorik im Deutschen Bundestag », Publizistik 7, (4), p.278-292.

Sarcinelli, U. (1992), « ‘Staatsrepräsentation' als Problem politischer Alltagskommunikation :

Politische Symbolik und symbolische Politik » in : Gauger, J.-D./Stagl, J. (éd.) : Staatsrepräsentation, Berlin : Reimer, p. 159-174

Sarcinelli, U. (1998) (éd.), Politikvermittlung und Demokratie in der Mediengesellschaft, Bonn :

Bundeszentrale für politische Bildung.

Scheuch, E. K./ Scheuch, U. (1992), Cliquen, Klüngel und Karrieren, Reinbek : Rowohlt.

Schütz, A. (1971), « Symbol, Wirklichkeit und Gesellschaft », in Id. : Gesammelte Aufsätze I, La Haye : Nijhoff, p. 331-411.

Schütz, A. (1972), « Santayana über Gesellschaft und Regierung », in Id. : Gesammelte Aufsätze 2, La Haye : Nijhoff, p. 174-202

Schütz, A. (1974), Der sinnhafte Aufbau der sozialen Welt, Francfort-sur-le-Main : Suhrkamp. 
Schütz, A. / Luckman, T. (1979,1984), Strukturen der Lebenswelt, 1 \& 2, Francfort-sur-le-Main : Suhrkamp.

Schwab-Trapp, M. (2002), Kriegsdiskurse, Opladen : Leske + Budrich.

Schwartzenberg, R.-G. (1980), Politik als Showgeschäft, Düsseldorf/Vienne : Econ.

Scott, M. B./ Lyman, S. M. (1976), « Praktische Erklärungen » In: Anwärter, M./ Kirsch, E./ Schröter, K. (éd.) : Seminar: Kommunikation, Interaktion, Identität, Francfort-sur-le-Main :

Suhrkamp.

Simmel, G. (1968), « Zur Philosophie des Schauspielers », in Id. : Das individuelle Gesetz, Francfortsur-le-Main : Suhrkamp.

Soeffner, H.-G. (1992), « Geborgtes Charisma - Populistische Inszenierungen » in Id. : Die Ordnung der Rituale, Francfort-sur-le-Main : Suhrkamp, p. 177-202.

Soeffner, H.-G. (1994), « Populisten: Profiteure, Handelsagenten und Schausteller ihrer Gesellschaften » in : Berking, H./ Hitzler, R./ Neckel, S. (éd.) : Politikertypen in Europa, Francfortsur-le-Main : Fischer, p. 259-279.

Sollmann, U. (1999), Schaulauf der Mächtigen, Munich : Knaur.

Tänzler, D. (2000), « Wem sind Politiker verpflichtet? » in : Meuser, M./Pfadenhauer, M. (éd.), Im Dienste der Menschheit? (Dokumentation des 5. Workshops des AK'Professionelles Handeln'): http://www.hitzler-soziologie.de/vernetzung.html

Vidal, G. (1973), « Barry Goldwater : A Chat », in Id. : Hommage to Daniel Shay : Collected Essays 1952-1972, New York : Random House.

Voegelin, E. (1965), Die neue Wissenschaft der Politik, Munich : Pustet.

Voegelin, E. (1996), Die politische Religionen, Munich : Fink.

Weber, M. (1980), « Politik als Beruf », in Id. : Gesammelte Politische Schriften, Tübingen : Mohr (Siebeck), p. 505-560.

Weiß, J. (1984), « Stellvertretung », Kölner Zeitschrift für Soziologie und Sozialpsychologie, 1, p. 43-55.

Wilson, F. G. (1936), The Elements of Modern Politics, New York : MacGraw-Hill.

Zimmermann, H.-D. (1969), Die politische Rede, Stuttgart : Kohlhammer.

\section{NOTES}

1. Voir Price/Bell (1970).

2. Sur cet ensemble de problèmes, voir également Tänzler (2000).

3. Voir Hitzler (1996).

4. Heller (1982), p. 388. Avec moins de scrupules que Hans-Georg Soeffner (1992), par exemple, qui souligne qu'il convient de faire une différence entre le charisme politique et le populisme pur et simple, et qui rattache les effets charismatiques à des éléments de représentation rituels particuliers (voir Soeffner [1994]), ou que Wolfgang Lipp, selon qui le charisme résulte de la capacité à assumer des risques vitaux ou des actes de stigmatisation sociale, j'entends ici par importance charismatique 'uniquement' la reconnaissance d'une compétence (ou de compétences) par d'autres qui leur font apparaître l'exercice d'un pouvoir sur eux-mêmes (ou sur des tiers) comme acceptable ou même souhaitable. 
5. Voir Laswell (1951).

6. Voir. Laswell (1954)

7. Renate Mayntz et Fritz Scharpf (Mayntz/Scharpf [1973], p. 121) parlent dans ce contexte d'une « contrainte institutionnelle à la réussite à court terme ». En effet, puisqu'on a tantôt « besoin » de tel ou tel et avant tout parce que l'on se sait jamais si l'on n'aura pas « besoin » de tel ou tel une nouvelle fois, en tant qu'homme qui ne vit pas seulement pour la politique, mais qui vit ou doit vivre de la politique en tant que métier, on a tout intérêt à garder ses propres ambitions aussi flexibles que possibles, ou tout du moins à les formuler de manière aussi flexible que possible (voir Hoffmann [1982]). Le politicien professionnel apparaît ainsi (ici et) aujourd'hui symptomatiquement comme un « gestionnaire des nécessités » tributaire de certains intérêts et privé d'indépendance, qui se réunit avec d'autres mandataires liés par des consignes pour faire entériner des décisions qui ont été prises ailleurs (voir Leibholz [1958]). Il se comporte donc volontiers, du moins à l'égard de ses électeurs et de ceux qui le soutiennent, comme un « agent de change social » (voir Boissevain [1978]) : il promet et distribue de préférence des ressources provenant « des poches de quelqu'un d'autre ", qui peuvent être publiques, mais aussi privées. Le politicien professionnel s'est d'ailleurs manifestement « spécialisé dans la légitimation de répercussions qu'il n'a pas causées et qu'il n'est pas non plus réellement en mesure d'éviter. » (Beck [1986]

p. 143.)

8. Vidal (1973).

9. Krockow (1989), p. 9.

10. Machiavel (1972), p. 73.

11. Max Weber (1980), p. 506.

12. Même les « députés d'arrière-ban » et les « souris grises » du Parlement doivent disposer de cette compétence (celle de convaincre les autres à leur sujet, ou de les convaincre que comparativement, ils ne peuvent faire de meilleur choix), faute de quoi ils n'auraient pas été élus députés. Qu'ils deviennent des « députés d'arrière-ban » et des « souris grises » est précisément dû à leur incompétence relative (en comparaison d'autres parlementaires) à donner une impression de compétence.

13. Voir Dörner (2001) ; Meyer (1992) ; et Schwarzenberg (1980).

14. Voir Goffman (1969).

15. Voir Pesch (2000).

16. Simmel (1968), p. 79

17. Freyer (1986), p. 50.

18. Voir Leif (2001), Jarren (2001).

19. Luckmann (1985).

20. Voir Berger/Luckmann (1969), Scott/Lyman (1976).

21. Voir Leif/Legrand/Klein (1992), Beyme (1993).

22. Voir Hitzler (1994).

23. Une réponse à cette question est peut-être que les hommes politiques doivent d'une part constamment convaincre qu'ils ont des compétences spécialisées sur des questions (manifestement) épineuses pour justifier toute "prétention à gouverner » (et les privilèges qui en résultent) mais qu'ils doivent aussi constamment, par ailleurs, convaincre qu'ils ne s'accordent ni ne veulent s'accorder aucune espèce de «droits spéciaux ». Voir Mayntz/ Neidhardt (1989).

24. À titre d'exemple : Edelmann (1988).

25. À mon avis, et justement d'une perspective dramatologique, il n'y a pas grand sens à hypostasier quelque mystérieuse «coulisse » où se ferait la politique réelle, tandis que seule la 
«fallacieuse apparence» serait présentée sur le devant de la scène. La politique a lieu en réalité sous de nombreuses formes, sur des scènes très diverses et dans toutes sortes de coulisses, avec des acteurs dont les talents, les dispositions et les engagements sont très divers. La lutte sur la scène médiatique publique est une part plus essentielle et plus évidente, même aux yeux de «l'homme de la rue », de la lutte pour le pouvoir, et celle-ci est souvent aussi une lutte pour la faveur du public (Ce n'est pas uniquement que la popularité ait l'avantage de permettre de parvenir au pouvoir, c'est aussi que le pouvoir a l'avantage de permettre de devenir populaire.). C'est pourquoi, selon moi, les mêmes règles dramaturgiques sont en vigueur dans toutes les situations où l'on «fait» de la politique (puisque la politique commence précisément sitôt qu'existe un espace public, de quelque nature qu'il soit.)

26. Ce qui est visible avant tout, ce sont les mesures de communication publique, d' «explication» et de justification, donc la présentation dite politique d'événements, de processus et d'état de choses (voir Nedelmann, 1986). Cela commence avec les interjections "spontanées " consignées dans les compte-rendus de séances et est très loin de trouver son terme dans les discours télévisés.

27. Par exemple Atkinson (1984), Graber (1976), Dieckmann (1969), Dörner (2001), Geißner (1969), Haseloff (1969), Sandow (1962), Zimmerman (1969).

28. Sarcinelli (1992), p. 165. Voir également Sarcinelli (1998).

29. Voir Dörner (2000). Il n'est aujourd'hui pratiquement aucune particularité, aucune singularité, aucun attribut, aucun accessoire de l'homme politique qui ne puissent, par l'entremise des caméras de télévision, être mis au centre de l'intérêt du spectateur (une remarque que l'on trouve déjà chez McGinnis 1970). En bref : les effets de la communication non verbale sont aujourd'hui au moins aussi importants - aux yeux de certains : bien plus importants - que n'importe quel contenu exprimé, du moins par le biais d' un écran. Ou pour le dire de manière moins formelle : le "message » politique perd une considérable part de son impact si le contenu, les intonations et l'apparence extérieure ne se renforcent pas mutuellement.Voir Sollmann (1999).

30. Voir Goffman (1971), Boorstin (1987.).

31. Voir Burke (1945).

32. Voir Schütz/Luckmann (1979), p. 139 sq.

33. Voir par exemple Radunski (1980).

34. Néanmoins, dans la mesure où le citoyen normal n'a jamais l'occasion d'appréhender cette communauté politique, pas plus que sa volonté générale, le «fonctionnement» d'un représentant politique exige qu'il soit ancré dans un schéma d'interprétation connu et compréhensible pour l'essentiel par les participants, qu'ils soient réceptifs ou productifs, passifs ou actifs. Ce schéma nous est transmis socialement par tout ce que l'on considère d'une manière générale comme appartenant à la formation de la conscience citoyenne ou critique de la politique (et par conséquent aux connaissances-repères dans la politique, voir Hitzler [1997]): de l'éducation civique à l'école, des ouvrages explicatifs et didactiques, des initiatives de propagande des partis, églises, associations, mouvements sociaux de toutes sortes, jusqu'aux informations politiques, commentaires et pamphlets; on doit aussi ajouter les potins politiques, la discussion de bistrot qui entretient le climat et les convictions politiques, tout comme les cercles de débat ou de contestation intellectualisés et plus ou moins subversifs.

35. Ferber (1970), p. 65.

36. Cette notion ne me paraît pas très éloignée du « Kosmion » d'Eric Voegelin (Voegelin [1965] ; Voegelin [1996]).

37. Selon Max Weber, le représentant est celui dont les actes peuvent être imputés à ceux qu'ils représentent, ou dont ces derniers reconnaissent qu'ils les engagent. Pour Francis G. Wilson, le représentant politique est le porte-parole de la volonté générale d'une communauté politique.

38. Voir Hitzler/Kliche (1995). 
39. Schütz (1972), p. 197.

40. Landshut (1964), p. 181-182.

41. Voir Schütz (1971), p. 409 sq.

42. Voir Rapp (1973), p. 133 sq.

43. Machiavel (1972), p. 72.

44. Gustav Ichheiser avait déjà fait observer que la "réussite » est essentiellement lié au fait de s'écarter des voies traditionnelles. La politique doit donc avant tout être comprise comme «la technique de l'agir social sous l'aspect de la réussite. » (Icheiser [1927], p. 300). À cette technique appartient la conscience que l'apparence de la vertu, et non la vertu réelle, est profitable aux buts politiques que l'on poursuit, parce qu'il est « de l'essence du pouvoir de se dissimuler sous le masque de la morale pour ne pas apparaittre comme ce qu'il est. » (Icheiser [1927], p. 309).

45. Machiavel (1972), p. 10. Pour un homme politique, le succès implique donc de s'orienter de facto moins en fonction de ce qui devrait être que de ce qui est effectivement, autrement dit d'agir indépendamment de l'idéologie et sans illusions (sur soi-même comme sur les autres). Pour Machiavel (et d'autres), le bon politicien n'est donc pas celui dont l'action est animée de bonnes intentions, mais celui qui fait bien ce qui doit être fait (pour le bien de la nation), parce que, comme nous le savons au moins depuis Foucault (Foucault 1978), le "pouvoir » et la « violence » ne disparaissent nullement de la société du simple fait qu'il est devenu plus difficile d'identifier distinctement ceux qui en sont les porteurs. La violence, comprise indépendamment de tout jugement de valeur comme une activité qui revêt des formes multiples (Voir Hitzler [1999]) est toujours l'ultima ratio lorsqu'il s'agit d'imposer des visées de pouvoir et de domination, telles qu'elles sont plus ou moins le monopole - du moins revendiqué - de l'État.

46. Landshut (1964), p. 181.

47. Pour un panorama de la théorie de la représentation récente, dans une acception empirique, voir Patzelt (1991).

48. À ce sujet, voir Berger/Luckmann (1969), p. 112 sq.

49. Husserl (1970), p. 340-373.

50. Voir. Schütz (1971) ; Schütz (1974), p. 165 sq. ; Schütz/Luckmann (1984), p. 178-200.

51. Voir Schütz/Luckmann (1984) , p. 364 sq. ; Schütz (1971)

52. Voir Eulau/Karps (1977).

53. Voir Schütz (1974), p. 172 sq.

54. Cité d'après Marx/Pankoke (1992), p. 97.

55. Voir Weiß (1984) ; Bourdieu (1986) ; Tänzler (2000).

56. Voir Schwab-Trapp (2002).

57. Voir Scheuch/Scheuch (1992) ; Arnim (1993).

\section{INDEX}

Mots-clés : fr

Schlüsselwörter : Repräsentation, Berufspolitiker 


\section{AUTEURS}

\section{RONALD HITZLER}

Ronald Hitzler ist professeur de sociologie générale à l'Université technique de Dortmund. Pour plus d'information, voir la notice suivante. 